\title{
STATIC RESPONSE OF THERMALLY LOADED SANDWICH BEAMS WITH CONFINED HORIZONTAL DISPLACEMENTS OF FACES AT THE SUPPORTS
}

\author{
Jolanta Pozorska ${ }^{1}$, Zbigniew Pozorski ${ }^{2}$ \\ ${ }^{1}$ Institute of Mathematics, Czestochowa University of Technology, Poland \\ ${ }^{2}$ Institute of Structural Engineering, Poznan University of Technology, Poland \\ Ijolanta.pozorska@im.pcz.pl, ${ }^{2}$ zbigniew.pozorski@put.poznan.pl
}

\begin{abstract}
The paper examines the problem of the influence of restrictions on freedom of horizontal displacements in thermally loaded sandwich beams. The classical theory of sandwich beams and panels with thin facings and a soft core has been applied. It was assumed that the cross-section of the beam could be asymmetric geometrically and materially. The beam has vertical supports and additional horizontal supports limiting the freedom of horizontal displacements. The supports are linearly elastic and the rigidity of the upper and lower support can be arbitrary. The static problem of single-span beam with support conditions identical at both ends of the beam was solved in the paper. Each of the sandwich facings has been subjected to temperature change. The derived formulas were used in the example illustrating the importance of the problem.
\end{abstract}

Keywords: sandwich panels, thermal actions, boundary conditions, elastic supports

\section{Introduction}

Due to their specific structure, sandwich panels used in civil engineering are characterized by relatively high load-capacity at low weight. Sandwich core made of polyurethane foam, mineral wool or polystyrene is an excellent thermal insulation. The core separates the faces, which results in a huge increase in the rigidity of the sandwich structure. Because during the operation of the building each external face of a sandwich has a different temperature, thermal stress has a huge impact on stress, strain and displacement of the panel.

In the generally accepted theory [1-4] structural behavior of sandwich beams is described by ordinary differential equations. Relatively simple boundary conditions are used. In fact, beams and panels are spatial systems, which have much more complex support conditions. These conditions may be different for the lower and upper faces of the sandwich panel. Complex conditions can be taken into account by the theories of higher order [5-7], but their use requires sophisticated computational tools.

The problem of the proper boundary conditions is particularly important in the case of thermal actions. The thermal actions are distortions. The influence of the initial distortions on the optimal design of support conditions in beams and frames 
was studied in [8]. In the case of statically undetermined systems, deformations induced by temperature are constrained. The result is a corresponding state of stress. In [9] it is shown that the stress caused by temperature occurs in the singlespan beam with a deep-profiled facing. It was shown in [10] and [11] that the sandwich structures are very sensitive to the change of the support conditions.

This paper proposes taking into account possibility of limiting the freedom of horizontal displacements at the supports. The formulas for the support reactions and stress in the faces were derived. On this basis, it is relatively easy to determine the appropriate field of strain and displacements. Presented in the article example illustrates the importance of this phenomenon. This work is a continuation of [12], which considered only systems that were symmetrical geometrically and materially.

\section{Formulation of the problem}

Consider a sandwich beam with thin but rigid facings and a thick, soft core. Action of static thermal load is examined. The beam's initial temperature is $T_{0}$. In the steady state, the upper face has a temperature $T_{1}$, while the lower face has a temperature $T_{2}$. In this paper we consider a sandwich beam with the boundary conditions shown in Figure 1. Extension or contraction of faces is limited by spring supports with stiffness $k_{1}$ (upper face) and $k_{2}$ (lower face). We allow all extreme cases such as rigid support $\left(k_{1}=\propto, k_{2}=\propto\right)$, the freedom of displacement $\left(k_{1}=0\right.$, $\left.k_{2}=0\right)$ and others. We assume different thickness of faces $\left(t_{1}-\right.$ thickness of the upper face, $t_{2}$ - thickness of the lower face), various modules of elasticity $\left(E_{1}, E_{2}\right)$ and different coefficients of thermal expansion of the face materials $\left(\alpha_{1}, \alpha_{2}\right)$. Unknown horizontal displacement $u$ and vertical displacement $w$ are functions of the variable $x$ determining the position of a point along the beam length.

a)

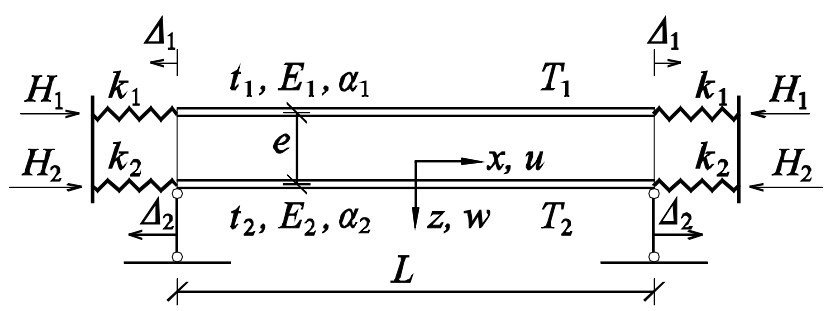

b)

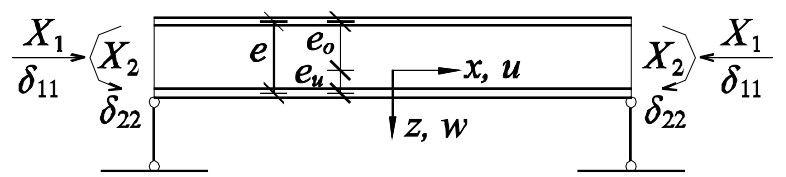

Fig. 1. The scheme of a one-span system with limits of horizontal displacements: a) boundary conditions and material parameters of the structure, b) unknown support reactions and displacements 
The core provides cooperation of faces, however, due to its material parameters (longitudinal elasticity module is about 50,000 times smaller than the modulus of steel), the normal stresses in the core can be ignored. It should however take into account shear deformation of the core.

We are looking for a solution of the single-span beam with the same supports at both ends (Fig. 1). The resulting solution will be able to be generalized to even more difficult, but a little practical case of multi-span beam with different spans and different stiffness of each elastic support.

\section{Solution of the static problem with thermal action}

Consider the beam presented in Figure 1 that originally had a temperature $T_{0}$. After some time, the upper and lower faces have reached temperatures $T_{1}$ and $T_{2}$. The change in temperature is a distortion. If it were possible, the upper and lower faces would extend in the longitudinal direction $\varepsilon_{1}=\alpha_{1}\left(T_{1}-T_{0}\right), \varepsilon_{2}=\alpha_{2}\left(T_{2}-T_{0}\right)$, respectively. Because the horizontal displacements are limited, such displacement field is kinematically inadmissible. Thus, there is a certain stress field arising from the supports interaction. One of the key issues in the analysis of systems with a non-symmetric cross-section is to determine the axis of the beam. The neutral axis of the beam is best to designate so that longitudinal strain did not induce bending moments and the curvature did not cause the normal force. In the case of crosssection composed of materials having different longitudinal elasticity module, the distance from the beam axis to the axis of the upper face $e_{o}$ and to the axis of the lower face $e_{u}$ is:

$$
\begin{aligned}
& e_{o}=\frac{E_{2} t_{2}}{E_{1} t_{1}+E_{2} t_{2}} e, \\
& e_{u}=\frac{E_{1} t_{1}}{E_{1} t_{1}+E_{2} t_{2}} e,
\end{aligned}
$$

where $e$ is the distance between axes of faces. For such determined axis of the beam, the bending stiffness is:

$$
B_{S}=\frac{E_{1} t_{1} E_{2} t_{2} b}{E_{1} t_{1}+E_{2} t_{2}} e^{2}
$$

where $b$ denotes width of the beam. It is worth noting that in the classical theory of composites, it is assumed that the axis of the composite is in the middle of its height and generalized strain and stress fields couple.

The issue of thermal loads requires some discussion. In the case of beams with a symmetrical cross-section thermal effects are usually separated into uniform and 
non-uniform parts. The first part causes extension or contraction of the whole element, while the second part induces curvature. In the case of non-symmetrical cross-sections the partition is not desirable because the thermal expansion coefficients of face materials are not in any relationship to the Young modules. As a result, the partition of the thermal effect on uniform and non-uniform may complicate rather than simplify the problem. It is worth noting that the correctly specified curvature (in some publications curvature is formulated incorrectly) induced by the change of the temperature in the faces, has the following form:

$$
\theta=\frac{\alpha_{2}\left(T_{2}-T_{0}\right)-\alpha_{1}\left(T_{1}-T_{0}\right)}{e} .
$$

A single-span beam with horizontal spring supports limiting horizontal displacements is shown in Figure 1a. Thermal actions induce forces $H_{1}$ and $H_{2}$ in the elastic supports. Both these forces, as well as other static quantities, depend on the Young modules, thermal expansion coefficients of facing material, thickness of faces, the distance between the facings (beam height), the width $b$ and the span $L$ of the beam and load conditions - temperatures $T_{0}, T_{1}$ and $T_{2}$. Unknown horizontal displacements of the upper and lower face are denoted as $\Delta_{1}$ and $\Delta_{2}$. Unknown forces $H_{1}$ and $H_{2}$ can be expressed by unknown axial force $X_{1}$ and bending moment $X_{2}$ (Fig. 1b):

$$
\begin{aligned}
& H_{1}=X_{1} \frac{E_{1} t_{1}}{E_{1} t_{1}+E_{2} t_{2}}-\frac{X_{2}}{e}, \\
& H_{2}=X_{1} \frac{E_{2} t_{2}}{E_{1} t_{1}+E_{2} t_{2}}+\frac{X_{2}}{e},
\end{aligned}
$$

The forces $H_{1}$ and $H_{2}$ are proportional to the stiffness of the support $k_{1}, k_{2}$ and displacements $\Delta_{1}$ and $\Delta_{2}$. The horizontal displacements of the supports $\Delta_{1}$ and $\Delta_{2}$ depend on the forces $X_{1}, X_{2}$ and temperature changes in the faces:

$$
\begin{aligned}
& H_{1}=k_{1} \Delta_{1}=k_{1}\left[-X_{1} \delta_{11}+e_{o} X_{2} \delta_{22}+\delta_{1 T}\right], \\
& H_{2}=k_{2} \Delta_{2}=k_{2}\left[-X_{1} \delta_{11}-e_{u} X_{2} \delta_{22}+\delta_{2 T}\right] .
\end{aligned}
$$

The symbols used in formulas (9) and (10) denote: $\delta_{11}$ - the horizontal displacement caused by the virtual force $x_{1}=1, \delta_{22}$ - rotation caused by the virtual moment $x_{2}=1, \delta_{1 T}$ - horizontal displacement caused by the temperature difference $T_{1}-T_{0}, \delta_{2 T}$ - horizontal displacement caused by the temperature difference $T_{2}-T_{0}$. In the case of beams shown in Figure 1b, the corresponding displacements and rotations are expressed as follows: 


$$
\begin{gathered}
\delta_{11}=\frac{1}{b t_{1} E_{1}+b t_{2} E_{2}} \cdot \frac{L}{2}, \\
\delta_{22}=\frac{1}{B_{S}} \cdot \frac{L}{2}=\frac{E_{1} t_{1}+E_{2} t_{2}}{E_{1} t_{1} E_{2} t_{2} b e^{2}} \cdot \frac{L}{2}, \\
\delta_{1 T}=\alpha_{1}\left(T_{1}-T_{0}\right) \cdot \frac{L}{2}, \\
\delta_{2 T}=\alpha_{2}\left(T_{2}-T_{0}\right) \cdot \frac{L}{2} .
\end{gathered}
$$

Comparing the right hand-sides of equations (7), (8) to (9), (10) and substituting (11)-(14), we obtain $X_{1}$ and $X_{2}$. The expressions for $X_{1}$ and $X_{2}$ are complex and depend on the $k_{1}$ and $k_{2}$, as well as other parameters of the model. Using the formulas derived for $X_{1}$ and $X_{2}$, and using (7), (8) we obtain much simpler expressions for $H_{1}$ and $H_{2}$, and each of these depends only on the value of a respective support stiffness:

$$
\begin{gathered}
H_{1}=\frac{E_{1} t_{1} b k_{1} L}{2 E_{1} t_{1} b+k_{1} L} \alpha_{1}\left(T_{1}-T_{0}\right), \\
H_{2}=\frac{E_{2} t_{2} b k_{2} L}{2 E_{2} t_{2} b+k_{2} L} \alpha_{2}\left(T_{2}-T_{0}\right) .
\end{gathered}
$$

Knowing all the forces acting on the structure, we can readily determine the displacements, strain and stress.

\section{Assessment of the impact of restrictions on freedom of horizontal displacements on the behavior of sandwich structure}

To demonstrate the impact of restrictions on freedom of horizontal displacements of the sandwich panel on internal forces, a single-span beam shown in Figure 1a was considered. The following geometrical and material parameters were assumed: $L=3.0 \mathrm{~m}, \mathrm{~b}=1.0 \mathrm{~m}, e=0.0995 \mathrm{~m}, t_{1}=t_{2}=0.0005 \mathrm{~m}, E_{1}=E_{2}=$ $=210 \mathrm{GPa}, \alpha_{1}=\alpha_{2}=12 \cdot 10^{-6} 1 /{ }^{\circ} \mathrm{C}$. Thermal conditions represent typical conditions in the summer: $T_{1}=65^{\circ} \mathrm{C}, T_{2}=20^{\circ} \mathrm{C}$. Installation temperature of sandwich panels was $T_{0}=10^{\circ} \mathrm{C}$.

Figure 2a shows the stresses in the faces as a function of the stiffness of the support $k_{1}$, for fixed $k_{2}=1 \mathrm{GN} / \mathrm{m}$ (support practically unmovable). In Figure $2 \mathrm{~b}$ the stresses in faces as the function of $k_{2}$, for fixed $k_{1}=1 \mathrm{GN} / \mathrm{m}$ can be observed. 
a)

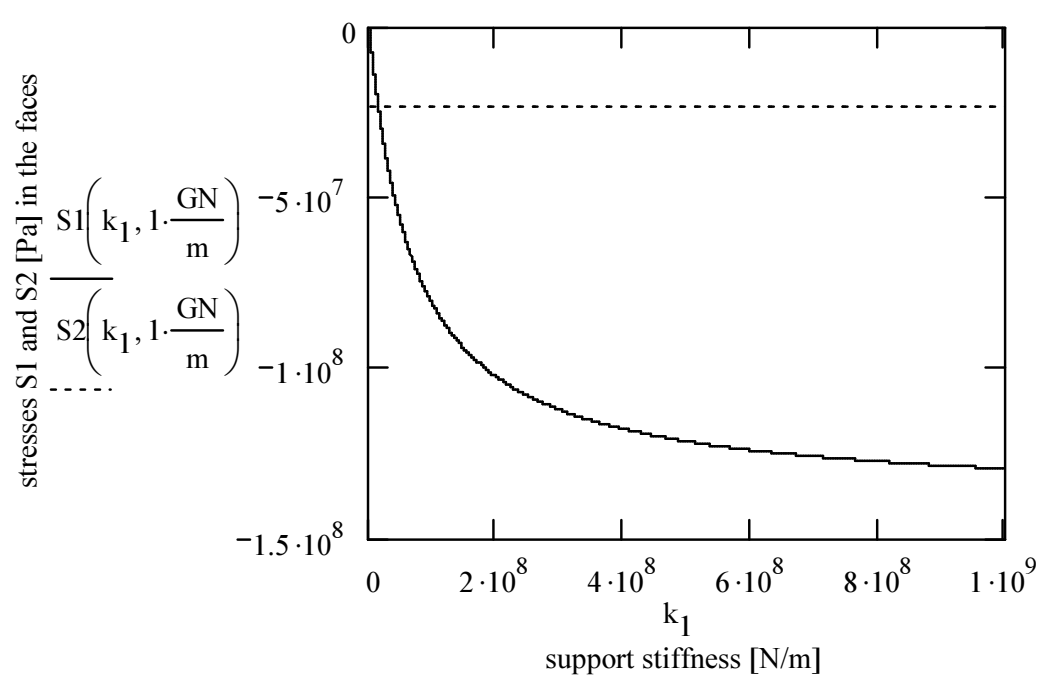

b)

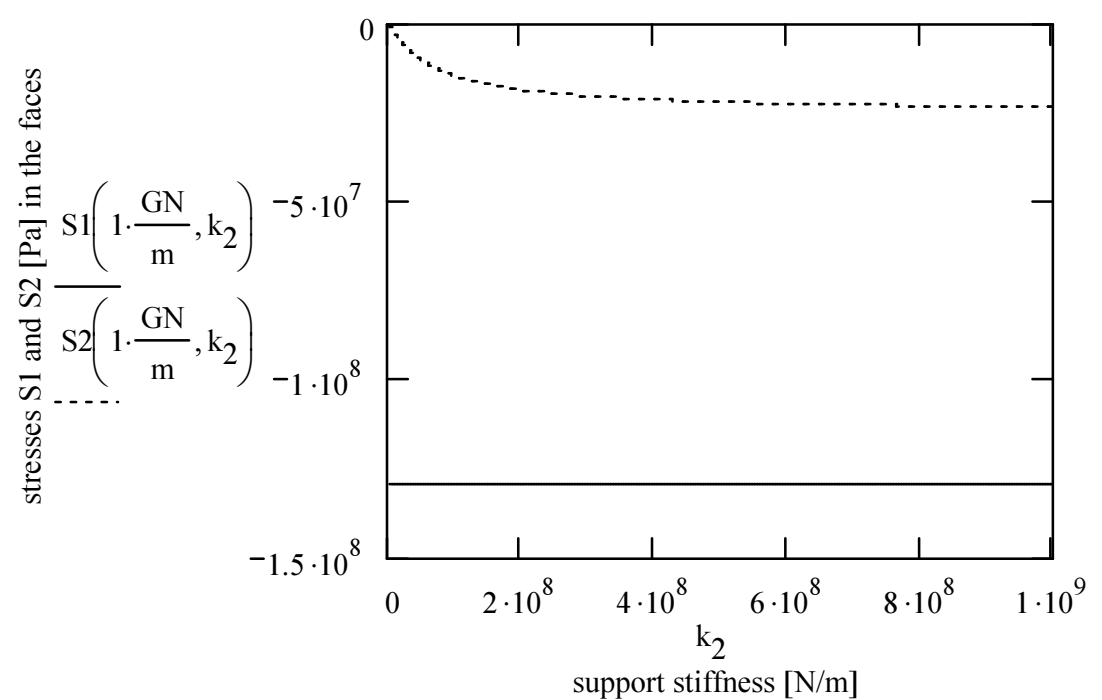

Fig. 2. The stress level in the upper face (S1 - solid line) and in the lower face (S2 - dashed line) as the function of stiffness coefficients of the horizontal supports: a) fixed $k_{2}=1 \mathrm{GN} / \mathrm{m}$ and variable $k_{1}$, b) fixed $k_{1}=1 \mathrm{GN} / \mathrm{m}$ and variable $k_{2}$

Discussing the obtained results, it can be concluded that the limiting of horizontal displacements causes high stresses. With the assumed thermal conditions they reach for the upper and lower facing respectively -129.5 and $-23.55 \mathrm{MPa}$, respectively. The analysis of formulas (15) and (16) shows that the stresses in the facings will be the greater, the higher the coefficient of thermal expansion and the temperature difference between the current and the reference temperature (temperature of assembly). In addition, an increase in the length of the beam, the 
stiffness of the support or the Young module of face material practically increases the stress in the faces. Fortunately, the actual structures are flexible. The conducted analyzes show that in the case of sandwich panels, the rigidity of horizontal supporting is about $10000 \mathrm{kN} / \mathrm{m}$. In this case, the stresses in the facings are falling radically.

\section{Conclusions}

The derived formulas and obtained results illustrate the impact of restrictions on freedom of horizontal support displacements on the behavior of beams and plates. The equations presented in the work are valid for non-symmetrical cross-sections of beams, for the full range of support elasticity. These formulas can be extended to the case of multi-span panels with arbitrary support conditions.

The presented example proved that in the case of full restriction on horizontal displacement, the stresses in the faces $100 \mathrm{MPa}$. The fact that the temperature at the assembly affects the values of these stresses is also noteworthy. With a susceptibility that exists in the real structures, stresses caused by the limitation of horizontal displacements are not so high, however, the horizontal forces affects the mechanical fasteners. Moreover, the effect of temperature causes extension, contraction and bending of the sandwich panel. All these aspects should be taken into account in the design of the building envelope made of sandwich panels.

\section{References}

[1] Davies J.M., Lightweight Sandwich Construction, Blackwell Science Ltd., Oxford 2001.

[2] Stamm K., Witte H., Sandwichkonstruktionen, Berechnung, Fertigung, Ausführung, SpringerVerlag, Wien 1974.

[3] Zenkert D., An Introduction to Sandwich Construction, Engineering Materials Advisory Services Limited, London 1995.

[4] EN 14509:2010 Self-supporting double skin metal faced insulating panels - Factory made products - Specifications, 2010.

[5] Frostig Y., High-order bending of sandwich beams with transversely flexible core and unsymmetrical composite skins, Composite Engineering 1995, 5(4), 405-414.

[6] Brischetto S., Carrera E., Demasi L., Improved bending analysis of sandwich plates using a zigzag function, Composite Structures 2009, 89, 408-415.

[7] Lezgy-Nazargah M., Shariyat M., Beheshti-Aval S.B., A refined high-order global-local theory for finite element bending and vibration analyses of laminated composite beams, Acta Mechanica, 2011, March, 217, 3-4, 219-242.

[8] Garstecki A., Mróz Z., Optimal design of supports of elastic structures subjected to loads and initial distortions, Journal of Structural Mechanics 1987, 15, 1, 47-68.

[9] Błaszczuk J., Pozorski Z., Engineering aspects of the mathematical model of deep-profiled sandwich panel, Scientific Research of the Institute of Mathematics and Computer Science 2011, 10(2), 31-42. 
[10] Studziński R., Pozorski Z., Garstecki A., Sensitivity analysis of sandwich beams and plates accounting for variable support conditions, Bulletin of the Polish Academy of Sciences - Technical Sciences 2013, 61, 1, 201-210.

[11] Sokolinsky V., Frostig Y., Boundary condition effects in buckling of soft core sandwich panels, J. Eng. Mech. 1999, 125(8), 865-874.

[12] Błaszczuk J., Pozorski Z., The influence of thermal actions and complex support conditions on the mechanical state of sandwich structure, Journal of Applied Mathematics and Computational Mechanics 2013, 12(4), 13-21. 